\title{
DYNAMIQUE DES CLIMATS ET DE L'ÉCOULEMENT FLUVIAL
}

La description des milieux naturels du Globe par un examen des grands mécanismes climatiques régissant l'écoulement liquide à la surface de la Terre est une tentative qui vient d'être faite par R. FréCAUT et P. PAGNEY (1).

Fruit de la collaboration d'un hydrologue et d'un climatologiste, ce document de base, fondamental à toute étude géographique, est plus qu'un livre d'hydrologie géographique, plus qu'un ouvrage de climatologie. L'orientation est claire; il s'agit de replacer les modalités de l'écoulement fluvial dans leur cadre climatique, c'est à dire, dégager l'influence essentielle des facteurs du climat dans la genèse et la variabilité de l'écoulement de surface. C'est un objet tout à fait actuel qui concerne une grande part de la communauté scientifique et qui commence à préoccuper les pouvoirs publics ayant le devoir de gérer l'eau, la composante la plus importante de notre environnement naturel, jusqu’à présent si maltraitée.

Dire que l'eau joue un rôle irremplaçable dans la vie, l'activité et l'histoire de l'Homme, que sans elle, la vie sur la Terre ne serait pas possible (tout au moins sous la forme que nous lui connaissons) est devenu un lieu commun. Depuis une quinzaine d'années se sont intensifiées les recherches sur les bilans hydriques indissociables de celles sur les bilans energétiques de notre système climatique. La nótion de bilan d'eau est devenue familière aux géographes et le cycle hydrologique constitue le concept fondamental de l'hydrologie. Conçu comme une circulation fermée de phénomènes naturels en chaỉne qui chargent l'atmosphère en vapeur d'eau puis la restituent de nouveau à la Terre par la condensation et l'écoulement, le cycle de l'eau présente deux branches distinctes. Sur la branche atmosphérique prédominent les flux d'eau sous forme gazeuse; sur la branche terrestre, l'eau se présente. sous sa forme liquide ou solide (cryosphère). Le moteur du circuit est alimenté par la radiation solaire, la chaleur latente de condensation et l'action de la gravité (énergie potentielle). De par son action sur la branche aérienne, la circulation atmosphérique générale conditionne donc le cycle hydrologique $\left({ }^{2}\right)$. Par ce biais; force est de constater que la trame climatique du Globe guide le découpage hydrologique des conti-

(1) F RECAUT, R.; PAGNeY, P., Dynamique des climats et de l'écoulement fluvial, Paris, Mạsson, 1984, $239 \mathrm{p}$.

(2) Voir des travaux de J. PINTO PEIXOTO sur ce sujet, en particulier $O$ ciclo da água em escala global. Lisboa, Comissão Nacional do Ambiente, 1979, et Atmospheric Vapour Fluxes for Hydrological Purposes, WMO/IND. n. ${ }^{\circ} 20$, Genève, 1973. 
nents. La dynamique de l'écoulement fluvial est donc intimement liée à celle de la genèse et de la variabilité des climats.

Dans la première partie, au long de quatre chapitres, remarquables par leur clarté, P. PAGNEY rappelle au lecteur les principaux facteurs cosmiques et planétaires responsables de l'agencement zonal des climats et ceux de nature géographique qui morcellent plus ou moins cette disposition d'ensemble. De la superposition des effets des différents facteurs naít la mosaïque climatique du Globe. On y trouvera les fondements de la circulation atmosphérique générale envisagés à partir de relations systémiques simples. L'analyse systémique est encore peu employée chez les climatologues géographes français. Elle fournit pourtant un instrument logique très utile en géographie. Le climat est envisagé comme la réponse de l'état du système climatique (constitué par l'atmosphère, la lithosphère, la biosphère, la cryosphère, et l'océan) à un forcement externe continu et à l'ajustement à des transformations internes. La composante fondamentale du forcement externe est la radiation solaire qui se manifeste par l'existence de phénomènes cycliques qui interviennent dans les systèmes couplés océan-atmosphère et océan-lithosphère, notamment par des oscillations thermiques diurnes et saisonnières. On arrive ainsi á dégager l'intervention majeure des facteurs climatiques d'origine cosmique et planétaire dans la genèse et la dynamique de la zonation radiative (chap. II) et dans celles de sa corollaire, la zonation thermique de la Planète. L'équilibre radiatif constaté dans le système global, à l'échelle de l'année, malgré l'existence d'une source de chaleur aux basses latitudes et d'un puits énergétique aux latitudes élevées, implique des transferts méridiens de chaleur et de masse qui, du fait de la rotation de la Terre, se décomposent en un ensemble de circulations zonales. Ce sont des faits bien connus mais pas toujours expliqués de manière accessible et, surtout, sans l'apparat mathématique que le géographe est plus ou moins préparé à digérer. C'est ce qu'a réussi P. PAGNEY dans le chapitre II; chapitre qui ne manquera pas de rendre de précieux services aux étudiants et de les réconcilier avec ce point souvent épineux du programme de climatologie générale qu'est le fondement de la circulation atmosphérique, tout en leur donnant une bibliographie spécialisée très actualisée sur la question.

Dans le chapitre III, l'accent est mis sur les composantes géographiques du forcement interne du système climatique. Ce sont l'hétérogénéité de la surface terrestre, océans, continents, reliefs, végétation... autant de facteurs géographiques qui imposent des contraintes, des frontières mécaniques et thermodynamiques à l'atmosphère. Il en résulte une trame climatique complexe où "tantôt l'action des facteurs zonaux demeurent prioritaires, tantôt s'effacent devant l'effet majeur des facteurs géographiques " (p. 66). Ce sont surtout les fondements du découpage climatique qui sont livrés ici. Les climats du Globe, leur localisation, leurs caractères et les mécanismes auxquels ils sont associés sont longuement décrits et expliqués dans un ouvrage antérieur de P. PAGNEY (1976) ${ }^{3}$ ) où on peut déjà lire «la mosaïque climatique du Globe se présente... finalement comme le résultat d'un découpage, avec inclusion des divi-

(3) P. PAGneY, Les climats de la Terre, Paris, Masson, 1976, $150 \mathrm{p}$. 
sions d'une classe dans la classe supérieure... Or, tout ceci implique un certain équilibre. La mosäque climatique du Globe est effectivement en état d'équilibre mais celui-ci n'est pas immuable. La question qui se pose alors est celle de savoir si les réajustements sont forcément généraux ou s'il est possible de voir se développer, dans certains cas, des évènements purement régionaux». (ob. cit, 1976, p. 8). Question effectivement fondamentale si l'on songe que le stock d'eau circulant dans notre système climatique sous ses trois phases est intégralement conservé dans le cycle hydrologique. Des éléments de réponse sont apportés par le chapitre IV qui analyse la capacité d'adaptation de l'état du système à l'ajustement aux conditions nouvelles créés depuis la dernière glaciation, soit la dynamique de la mosaique climatique.

Ainsi, cette première partie qui constitue presque la moitié de l'ouvrage, fait-elle état des grands mécanismes climatiques qui régissent l'écoulement liquide à la surface de la Terre. Le chapitre $\mathrm{V}$ en constitue à la fois la conclusion et le point de dépàrt de l'hydrologue, R. FRECAUT, qui dégage l'influence prédominante des facteurs climatiques dans la genèse de l'écoulement fluvial et leur rôle varié selon les domaines climatiques considérés: influence déterminante des facteurs thermiques aux hautes et moyennes latitudes, rôle exclusif des facteurs pluviométriques aux basses latitudes.

Le cadre climatique ainsi posé, les processus d'écoulement sont analysés dans les deuxième et troisième parties de l'ouvrage, incluant la prise en compte, d'une part, des régions froides et tempérées et, d'autre part, des régions chaudes de basses latitudes. Le traitement des processus d'écoulement dans ce cadre essentiellement zonal, mais qui renferme de nombreux exemples régionaux, est particulièrement dynamique. Les grands mécanismes atmosphériques propres à chaque domaine sont exposés à grands traits et mis en relation avec les caractères originaux de l'écoulement liquide. La précision et l'abondance des données chiffrées, la qualité de la documentation, le recours à des travaux tant climatologiques que hydrologiques extrèmement récents donnent une exceptionnelle densité à ces deux dernières parties et témoignent de l'étroite collaboration qui existe depuis longtemps entre les deux auteurs.

Aux hautes latitudes, P. PAGNEY met l'accent sur l'importance des processus radiatifs dans l'originalité de la circulation polaire ainsi que sur la diversité régionale de ce monde polaire encore peu connu. Le froid quasi permanent exclut tout écoulement liquide aux très hautes latitudes, mais sur les marges polaires, à partir de travaux en cours, R. FRECAUT nous révèle le régime saisonnier extrêmement perturbé de l'écoulement calqué sur les saisons thermiques.

Les latitudes moyennes dominées par les transferts énergétiques, domaine d'affrontements atmosphériques et de systèmes tourbillonnaires, sont des régions source de condensations importantes. Cependant, les grands contrastes latitudinaux et régionaux comme la grande variabilité pluviométrique interannuelle, résultat de l'interdépendance des zones climatiques, en font un domaine climatique et hydrologique hétérogène. Dans les régions tempérées des latitudes moyennes, les facteurs thermiques modulent l'écoulement et les saisons hydrologiques sont encore intimement liées aux saisons thermiques, avec des périodes plus ou moins importantes de rétention nivale en fonction de la continentalité, de la latitude, de l'altitude... Neiges et glaciers 
constituent encore une part importante de l'alimentation fluviale. Les conditions thermiques constituent aussi le facteur essentiel de la régularité de l'alimentation fluviale: Elles en constituent en quelque sorte le régime moyen de l'écoulement. Les pluies, quant à elles, sont très variables d'une saison à l'autre, d'une année à l'autre. La fréquence d'une telle variabilité augmente vers la marge sud, en domaine méditerranéen. Les conséquences hydrologiques sont l'irrégularité interannuelle de l'abondance des cours d'eau, les gonflements exceptionnels des rivières calqués sur les épisodes pluvieux les plus longs ou les plus intenses et les étiages prolongés, reflet de l'intensité des périodes de sécheresse non seulement d'été mais aussi parfois hivernales. Au Portugal, nous connaissons bien les effets hydrologiques de l'inconstance du régime des pluies. Le monde méditerranéen, déjà subtropical, montre la transition vers les modalités d'écoulement des régions chaudes des basses latitudes en ce sens que le facteur climatique fondamental devient les précipitations pluvieuses.

Dans la troisième partie de l'ouvrage réservée aux régions chaudes, P. PAGNEY rappelle qu'entre les tropiques, on relève les espaces les plus secs du Globe et les territoires les plus arrosés. Ainsi sont mises en évidence d'une part, les régions source de vapeur d'eau que sont les anticyclones subtropicaux océaniques et, d'autre part, l'originalité des régions de convergence où cette vapeur d'eau est condensée. Ces mécanismes généraux mis en place et le facteur pluviométrique étant l'élément majeur de la différenciation climatique aux basses latitudes, le chapitre III donne le découpage régional. Les caractères régionaux et l'organisation des climats tiennent compte des saisons pluviométriques alternées (climats de mousson, d'alizé), des saisons sèches restreintes (climat guyanais) et des conditions extrêmes marquées par des pluies constantes ou une aridité quasi-permanente. Saisons pluviométriques et saisons hydrologiques sont intimement liées, parfois de manière tyrannique. En région chaude et sèche, à la limite, l'écoulement se calque sur l'averse si les facteurs orographiques et litho-pédologiques le permettent et il se confond avec la crue. La variabilité pluviométrique interannuelle augmente des régions équatoriales aux régions subtropicales et imprime son cachet sur l'abondance de l'écoulement. Ces variations dans l'abondance se marquent également par des crues brutales liées aux passages des systèmes très pluvieux (cyclones...); la sécheresse et les étiages corrélatifs existent même en régions équatoriales.

En somme, il s'agit d'un livre clair, agréable à consulter qui a l'avantage de donner, de forme concise, une vision globale de la dynamique des climats et de l'écoulement flụvial dans une perspective très actuelle. Il est simple car il ne présente que les grands traitș de l'écoulement, et parmi les facteurs physiques qui les régissent, surtout ceux d'origine climatique. Ainsi, volontairement, l'étude des grands organismes fluviaux à régimes complexes a été laissée de côté. Tous les liens unissant bilans d'énergie, bilans d'eau, mouvements de l'atmosphère et facteurs géographiques sont présentés de façon à éclairer la répartition logique zonale et régionale des climats, à montrer l'influence déterminante de certains éléments dans la modalité de l'écoulement et à souligner le caractère de sa variabilité. Bref, P. PAGNEY a tissé «le cadre à l'intérieur duquel l'hydrologue (R. FRECAUT) a choisi de poser certains problèmes et 
de situer certains aspects de l'écoulement à la surface de la Terre» (p. 218). Toutes les démonstrations s'appuient sur de nombreux exemples concrets et sur d'abondantes données chiffrées. La bibliographie climatologique et hydrologique tant française qu'étrangère est choisie et très récente. Ce sont près de 300 titres dont les $2 / 3$ sont postérieurs à 1975; un bon nombre d'entre eux se réfèrent à des travaux menés au Centre de Recherches de Climatologie de Dijon. En conclusion, c'est un livre sérieux qui amène une réelle contribution à la compréhension de la dynamique des milieux naturels. La présentation est à la hauteur de la réputation des éditions Masson. On peut regretter toutefois la trop grande sobriété de l'illustration et... pour nous, portugais, le coût élevé de l'ouvrage.

DENISE DE BRUM FERREIRA 